\title{
Seizure and Psychosis
}

\author{
A K Roy*, Rishabh Maloo, Saurabh Sadekar, Murali S and Vijayendra Reddy \\ Department of Neurology, Manipal Hospital, Bangalore, India
}

*Corresponding author: A K Roy, Department of Neurology, Manipal Hospital, HAL Airport Road, Bangalore, India

\begin{abstract}
Seizures are due to abnormal excessive and synchronous neuronal activity in the brain [1]. Outward effects vary from uncontrolled shaking movements involving much of the body with loss of consciousness, to shaking movements involving only part of the body with variable levels of consciousness, to a subtle momentary loss of awareness. Up to $10 \%$ of people have at least one epileptic seizure in their lifetime out of which $50 \%$ have a recurrence [2].
\end{abstract}

\section{Introduction}

\section{Aim}

To differentiate between different types of psychosis following epilepsy.

\section{Methods}

A prospective study was conducted including 1000 consecutive patients with seizure disorder irrespective of the seizure type and cause and followed up between 2010 to 2017. The clinical profile of patients with seizures with respect to age, sex, aetiology of seizure, neurological status, seizure type, investigations, prescription pattern of Anti-Epileptic drugs and response to treatment was analysed.

\section{Results}

Out of a total of 1000 patients, $59 \%$ of the population had focal seizures and $41 \%$ had generalised seizures, $66 \%$ were found to have an idiopathic onset, followed by neurocysticercosis, tuberculosis, vascular, post infective, post traumatic and congenital having $16 \%$, $8 \%, 3 \%, 3 \%$ and $2 \%$ of the cases respectively. In the study $45.77 \%$ of patients with focal seizures remained uncontrolled while $46.35 \%$ and $20 \%$ of tonic clonic and myoclonic seizures remained uncontrolled. A total of 430 patients were prescribed monotherapy while 460 were on two and 110 on 3 or more drugs. Thirty-eight patients had associated psychosis, 20 patients having postictal psychosis, 12 having brief psychosis out which 3 were chronic (lasting more than 6 months) and 6 having inter-ictal psychosis.

\section{Case Vignette}

A 79-yr old male known to have diabetes and hypertension was admitted with complaints of progressively increasing irritability, alteration in behaviour, forgetfulness and intermittent episodes of disorientation since last 2 weeks. His blood investigations showed hyponatremia of $118 \mathrm{meq} / \mathrm{L}$, rest of the blood investigations were within normal limits. Gradual hyponatremia correction was done over 2 days but there was no clinical improvement in the patients' clinical condition. Neuroimaging and CSF evaluation showed mild elevation in protein $(58 \mathrm{mg} / \mathrm{dl})$ with normal csf glucose, viral markers and autoimmune antibody panels. Possibility of psychosis due to prolonged hospitalisation was also considered and was started on haloperidol and quitiapine but did not cause any clinical benefit. His EEG showed attenuation of alpha rhythm corresponding to age. He was started on Inj Lamotrigine $100 \mathrm{mg}$ IV BD following which he showed improvement in the orientation and irritability over the next two days and was finally diagnosed as chronic ictal psychosis.

\section{Case II}

68-year-old lady admitted in an ICU at a hospital elsewhere 5 days back with accelerated hypertension, developed irritability, disorientation and loss of bowel and bladder control upon discharge the following day. Her blood investigations were normal including ammonia and liver function tests. Her MRI brain and CSF were within normal limits, EEG showed generalised slowing of 
theta- delta range. On day two an occasional jerky movement was noted over the face and started on Inj Lacosamide $100 \mathrm{mg}$ IV BD, she showed dramatic improvement after 12 hours, being able to eat solid food herself and walk independently by the evening. She was diagnosed to have brief psychosis with seizures.

\section{Discussion}

The association between epilepsy and psychosis has attracted the interest of neurologists and psychiatrists alike for many years. While much of this interest has concerned the chronic psychosis. The categorization of these brief psychotic syndromes has traditionally been according to their temporal relationship to the seizures, as ictal, postictal, and inter-ictal.

Psychosis: A psychiatric disorder characterized by delusions, hallucinations, disorganized speech or thought, and/or grossly disorganized or catatonic behaviour.

Brief Psychosis: Psychosis that lasts more than a day but generally less than 1 month. Some epilepsy-related brief psychoses may last up to 2-3 months. Psychoses lasting more than 6 months are chronic, and those lasting more than 3 months are tending to chronicity.

Postictal Psychosis: Psychosis that follows immediately after 1 or generally multiple seizures, but certainly within 1 week of the last seizure.

Inter-Ictal Psychosis: Psychosis that develops when the patient with epilepsy has not had a seizure for more than 1 week or is unrelated to the any recent increase in seizure activity [3] Psychosis may range from acute to subacute in presentation with clinical presentation ranging from mild disorientation to complete lack of awareness, being mute and unresponsive, frank catatonia, irritability and mania. Treatment would include primary biofeedback and behavioural therapy with pharmacotherapy mainly with atypical antipsychotics like quetiapine, olanzapine, aripiprazole. Initially to be started at a lower dosage of olanzapine $2.5 \mathrm{mg}$ daily to $5 \mathrm{mg}$ twice daily.

\section{Conclusion}

Though psychosis immediately following the episodes of seizures is most commonly encountered, chronic psychosis up to 6 months post the episode or those without any prior event are also present and should not be considered unrelated to the primary aetiology. Often it is encountered that a patient may present with psychosis and create a diagnostic dilemma due to long standing psychosis in a seizure disorder patient or psychosis presenting initially in a patient not having a prior history of evident seizures.

\section{References}

1. Melissa, Jasvinder Singh, Anthony Marson (2018) Epilepsy psychosis: a practical approach 18(2): 106-114.

2. Olsen T S (2013) Post-stroke epilepsy, pp. 340-344.

3. Kanemoto (2012) The advanced neurological disorder. Psychotic illness in epilepsy 5(6): 321-334.
(C) (P) This work is licensed under Creative Commons Attribution 4.0 License

DOI: $10.32474 /$ OJNBD.2019.02.000136

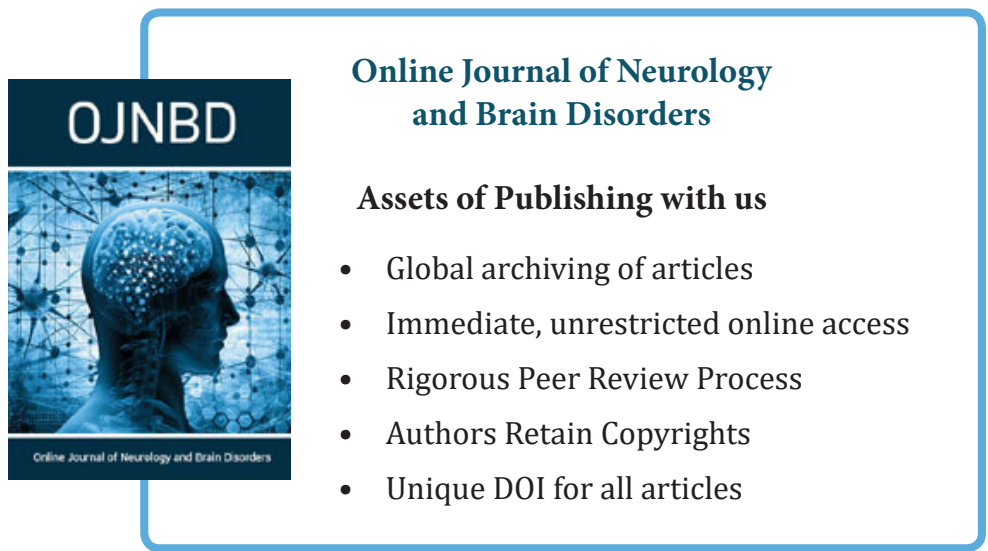

